\title{
LE GÉNIE DU FRANÇAIS TECHNIQUE
}

Claude Bédard

\section{INTRODUCTION}

Tout rédacteur technique francophone do1t être consclent que les moyens d'expression propres à la langue technique (et qu'il se doit d'acquérír avec le temps) diffèrent de ceux de la langue générale:

*Il y a d'abord et avant tout le vocabulaire spéclallsê (dynamomètre, thyristor, etc.), sans lequel on ne peut pas dire grand-chose en technique.

*I1 y a aussi les multiples expressions et tournures qu'on emplofe dans tel ou tel domaine technique pour enrober le vocabulaire spécialisé (la tension AUX BORNES DE la résistance...).

*Enfin, à un niveau plus global, 11 y a un certain nombre de tendances quí caractérisent 1a mantère d'écrire du locuteur technique. On pourra même parler de génie de la langue technique, différent à certalns égards de celui de la langue générale. C'est de ce génie souvent 1gnoré des rédacteurs techniques débutants qu'11 sera question 1 ci.

Le Québec n'ayant guère de tradition d'écriture technique (1'emplo1 du françals dans l'industrłe y date d'une vingtaine d'années), c'est dans les documents de France (livres et revues) que nous avons glané nos observations.

\section{LES TRAITS GÉNÉRAUX DE LA LANGUE TECHNIQUE}

Nous n'essaterons pas de classer les tratts observés dans les catégories lexicale, syntaxique et stylistique. Le praticien de 1 ' écriture ne tireralt guère profit d'une telle distinction, qui serait en outre artificielle dans blen des cas. Nous avons plutôt regroupé nos observations sous cinq grandes constatations.

LA LANGUE TECHNique EST IMAGËE

Sontrairement à une oplnion répandue, la langue technique est ioin d'être terne et sans vie. En falt, elle témolgne d'une grande vigueur qui se manffeste notamment par une foule d'emplois imagés. Ces Images sont très souvent un caractère animiste (on prête à des objets Inanimés des choses qui sont le propre des êtres vivants). Ces emplois se manifestent dans diverses catégories grammaticales:

\section{Substantifs}

La LONGÉvité d'un matérie1

La courbe de MORTALITÊ des lampes, leur ESPÉRANCE DE VIE

Le COMPORTEMENT, les PERFORMANCES d'un matérlel

L'AFFOLEMENT des soupapes d'un moteur

L'IMMNITÉ d'un matérlel électronique aux parasites

L'AGRESSIVITÉ chimique des produits pompés

Verbes

La courrole n'ADMET aucune varlation de longueur

Une soupape de sûreté CRACHE

Une courrole mal tendue CRIE, FOUETTE

Les bogles AVALENT les inégalités du sol

Le moteur REGIMBE à bas régime

DÉSHABILLER un arbre de boite de vitesses

La sortie de l'étage $X$ ATTAQUE 1 'étage amplificateur

Ces fonctions S'ACCOMODENT d'un certain désalignement

Adjectifs

Un plastique INDIFFÉRENT aux solvants

Le godet est ARMÉ de dents d'attaque

Un clapet FUYARD, un clapet DESEMPARE

Les poulles sont CHAUSSÉES d'une courrole crantée

Un matériel COMPATIBLE avec un autre

La dose d'animisme présente dans la langue technique s'explique peu être par le falt que les ouvriers et techniciens (et avant eux les artisans) vivent en contact étrolt avec les réalités matérielles; ayant l'esprit concret, 11 s se plaisent à des emplols qui font ima

Notons aussi que $I^{\prime}$ an1misme n'est nullement limité à des emplołs figés; en effet, de nombreux exemples relevés dénotent clairement que le locuteur crêe lui-même des animismes instantanés, indépendamment du réservoir d'emplois déjà en usage. 
LA LANGUE TECHNIQUE EST ÉCONOME

La langue technfque, souvent perçue comme lourde, recherche pourtant la brièveté par de multiples moyens: ellipse du déterminē, ellipse de la préposition, troncation, dérivation, etc. Cette tendance $s^{\prime}$ affirme de plus en plus nettement en langue technique moderne, au point qu'on peut dire sans risque que la langue technique est en avance sur la langue générale sur ce point.

\section{Ellipse du déterminé}

Ce procédé consiste à supprimer le premter mot du syntagme, souvent assez général pour ne pas être indispensable; le modificateur devient alors le mot principal. S'1l s'agit d'un

substantif, 11 passe généralement de l'abstrait au concret:
les () sécuritês
(dispositifs de)
un () verroulllage
(dispositif de)
les () étanchéités
(dispositifs $d^{\prime}$ )
équipés de () fins de course
(commandes de)

E1lipse de 1a préposition

Ce procédé consiste à faire sauter le mot-charnière (en génêral une préposition) qui explicite la relation entre deux substantifs d'un syntagme nominal:

$\begin{array}{ll}\text { alimentation () pompe principale (de la) } \\ \text { tube () acier gaíné () plastique (en) (de) } \\ \text { bilan () matières } & \text { (des) } \\ \text { sécurité () mémoire } & \text { (de la) } \\ \text { élévateur () potence } & \text { (en forme de) } \\ \text { compatible () TTL } & \text { (avec des composants) } \\ \text { pince gainée () confort } & \text { (pour assurer le) } \\ \text { polystyrène () choc } & \text { (résistant au) } \\ \text { cordon () secteur } & \text { (de raccordement au) }\end{array}$

Ce procédé a l'inconvénient de rendre le syntagme moins explicite. Par contre, 11 est un facteur incontestable de brièveté et de manlabllité; cela est d'autant plus apprécté que la majorité des termes nouveaux se présentent effectivement sous la forme de syntagmes nominaux.
Il s'agit là sans doute du trait le plus révélateur de l'évolution de la langue technique française: sacrifter quelque peu la clarté qu1 a fait sa grandeur pour deventr plus fonctionnelle. Et même si l'Influence de l'anglals n'est pas étrangère à certains emplois ( 1 ' exemple compatible TTL est un calque direct de TTL compatible), 11 nous semble que cette tendance procède d'une évolution authentiquement française.

\section{Troncation}

Ce procédé consiste à supprimer les dernières syllabes d'un mot. Par exemple, pneu(matique), auto(mobile). Il n'est pas récent, mais 11 tend, semble-t-1l, à sortir de l'argot d'atelfer pour entrer de plus en plus dans la langue technique de niveau correct:

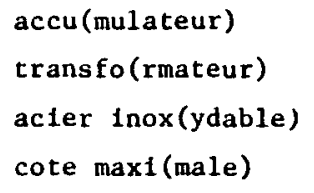

\section{Dérivation}

En français contemporain, la dérivation est au goût du jour. Et 11 semble que la langue technique soit encore moins réticente que la langue courante à former des dérivés. Nous ne chercherons pas à en présenter tci un éventail exhaustif, mals simplement à signaler les catégories suivantes, qui nous semblent les plus représentatives.

\section{Substantifs}

Les deux premières catégories nous semblent dériver non d'un phénomène rếcent, mais de la tradition du langage technique, héritée des artisans. La trołsième semble motivée par une tendance plus récente à l'intellectualisation:

\begin{tabular}{llll} 
Collectifs & Noms de Personnes & Caracteristiques \\
\cline { 2 - 3 } embiellage & caoutchoutier & & indesserrabilité \\
segmentation & raccordier & pelabilité \\
nervuration & pneumatiquier & Inoxydabilité \\
tôlerie & turbinier & malntenabilité \\
clouterle & clternfer & emboltabilité \\
pomperie & chaudiëriste & increvabilité \\
raccorderie & frigoriste & programmabilité
\end{tabular}


Signalons que les puristes reprochent aux substantifs en -abilité leur longueur, qui peut sembler abustve. Mals 11 est indéniable qu' 1ls répondent au désir d'un mot untque pré-emballé.

\section{Adjectifs}

La dérivation adjectivale a souvent 1'avantage de remplacer un groupe de mots (en gênéral un substantif complément et sa charnière) par un simple adjectif:

$\begin{array}{ll}\text { Adjectifs à source verbale: } & \\ \text { vis CALANTES d'un théodolite } & \text { (de calage) } \\ \text { pouvoir FILTRANT } & \text { (de filtration) } \\ \text { résines ÉCHANGEUSES d'ions } & \text { (d'échange) } \\ \text { témoin DÉTECTEUR } & \text { (de détection) } \\ \text { coffret PLOMBABLE } & \text { (pouvant être plombé) } \\ \text { filtre INENCRASSABLE } & \text { (ne s'encrassant pas) }\end{array}$

Sou1ignons la grande ut1litê des adjectifs en -able: outre 1'ut1lisation au sein d'un syntagme plus ou moins lexicalisé comme dans les exemples cltés, 11 y a l'emploi libre qui permet de remplacer une tournure plus lourde:

La vanne porte un chapeau (qui peut être remplacé)

remplaçable sans interruption de service.

Adjectifs à source nominale

$\begin{array}{ll}\text { tube AILETTÉ } & \text { (à ailettes) } \\ \text { fer à souder THERMOSTATÉ } & \text { (à thermostat) } \\ \text { signal BRUITÉ } & \text { (entaché de bruit) } \\ \text { roue JANTÉE de cuir } & \text { (à Jante de cuir) } \\ \text { lane PASTILLÉ de carbure } & \text { (à pastilles de carbure) } \\ \text { coussinets ANTIFRICTIONNÉS } & \text { (garnis de métal antifriction) } \\ \text { échangeur TUBULAIRE } & \text { (à tubes) } \\ \text { espace INTERDENTAIRE } & \text { (entre les dents) } \\ \text { perceuse MONOVITESSE } & \text { (à une vitesse) } \\ \text { câble MULTIBRINS } & \text { (à plusieurs brins) }\end{array}$

Signalons que l'adjectif à source nominale peut être quelque peu ambigu, à cause de la diversité des rapports nom/adjectif possibles. Par exemple, l'adjectif tubulaire (c1-dessus) peut signifier aussi bien a tubes qu'en forme de tube.
Le résultat n'est pas toujours plus court en nombre de syllabes; mais la compacité y gagne (1e nombre de mots diminue), ce qui semble être une des motivations à l'oeuvre dans la langue technique française.

\section{Verbes}

Cette catégorie est molns riche que les autres. On peut distinguer la formation:

A partir d'un verbe existant, par préfixation.

$\begin{array}{ll}\text { prélubrifier } & \text { désenfumer } \\ \text { relubrifier } & \text { déballaster }\end{array}$

Ce genre de dérivation, peu souvent consigné dans les dictionnatres, est souvent très courante et pratiquée librement.

A partir d'un substantff, par suffixation.

$\begin{array}{ll}\text { disjoncter } & \text { thermostater } \\ \text { clipser } & \text { électroniser } \\ \text { interfacer } & \text { robotiser }\end{array}$

Adverbes

L'emplo1 d'adverbes en -ment permet d'éviter des périphrases. La langue technique a donc tendance à former ce genre d'adverbe, surtout pour des situations courantes: par exemple axialement, radialement, et longitudinalement pour remplacer dans le sens axial...

Signalons aussi une tendance à afouter d'autres sens à des adverbes existants, ce qui bien entendu amène des ambiguités (un peu comme nous $I^{\prime}$ avons vu pour les adjectifs). Alnsi, l'adverbe électriquement, qui signifie normalement par des moyens électriques, est souvent employé pour signifier au point de vue électrique. Encore là, la brièveté semble voulolr l'emporter sur la clarté.

Pour terminer sur la dêrivation, on peut observer que les emplois cltés, pour la plupart, ne sont ni ne seront conslgnés dans les dictionnatres de langue: 11s sont trop nombreux et alourdiraient trop les dictionnatres; par allleurs, 1ls s'expliquent d'eux-mêmes en général.

Devant cette prolifération de dérivés, 11 faut constater que le locuteur (à l'instar des emplois imagés) se sent mantfestement libre de créer ses dérivés. A l'ère de la modularité, ces mots-constructions 
sont considérés comme simplement dispontbles à l'usager, prêts à être falts et défalts, sans qu'll fallle se poser la question de la mise au dictionnatre.

\section{Tours prépositifs économiques}

Certains tours prépositifs font sauter les articles (déftnis ou indéfinis); 11 en résulte une concision qui dégage du reste une certaine saveur intellectuelle:
EN fin de course
(à la fin de la)
EN bout de ligne
(au bout de la)

D'autres tours prépositffs permettent souvent l'ellipse plus payante d'un substantif. En particulier la préposition en, qui remplace elle-même des locutions prépositives:
EN basse tension
1 'ut 11 lsation EN voltmètre
(comme un)
1 'appareil fonctionne
utilisé EN chariot, le diable... (en tant que)
la pose des câbles EN encastré (de façon)

(dans le cas d'un circuit)

LA LANGUE TECHNIQUE CONNAITT LES NIVEAUX DE LANGUE

Il peut sembler curleux de volr aborder Icl une caractéristique qui n'est certes pas spécifique à la langue technique, mals qui est au contrafre une marque importante de la langue courante. Mais c'est justement parce qu'on ne pense génêralement pas à reconnaftre cette caractéristique à la langue technique que nous avons voulu en tralter 1c1.

L'aspect impressionnant du vocabulaire technique fait souvent penser aux débutants que ce vocabulaire n'a pas de nlveaux de langue. Or on peut distınguer trois palıers, qui s'ordonnent de façon parallèle à ceux de la langue courante, même si les êtiquettes diffèrent. On peut les rêsumer par le tableau suivant:

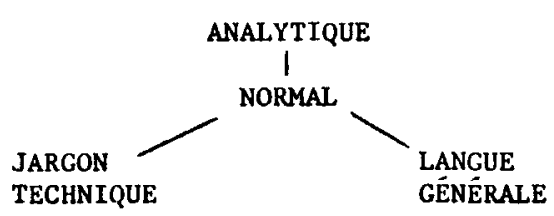

Le niveau analytique trouve son origine chez les chercheurs et les théoriciens.

Le niveau dit normal est celui du vocabulatre courant chez les utilisateurs des matérfels et des concepts mis au polnt par d'autres.

Au-dessous du niveau correct, on peut placer côte à côte le niveau du jargon d'atelier et celui des termes utilisés dans la langue générale pour désigner des réalités techniques.

\section{Volci quelques exemples:}

\begin{tabular}{|c|c|}
\hline Analytique & Correct \\
\hline accumulateur plomb-actde & batterie au plomb \\
\hline bandage pneumatique & pneu \\
\hline éclateur à sphères & éclateur à boules \\
\hline paller à charge axiale & butée, paller de butée \\
\hline paller à charge radiale & paller, palier porteur \\
\hline moteur à capsulisme & moteur thermique \\
\hline train épicyclordal & train planétalre \\
\hline Correct & Langue Générale \\
\hline lampe à Incandescence & ampoule électrique \\
\hline lampe fluorescente & néon \\
\hline roue dentée & plgnon \\
\hline
\end{tabular}

Il faut noter que c'est souvent au niveau analytique que se créent les génériques dont le vocabulaire technique a besoin. Exemples:

$$
\begin{aligned}
& \text { aéronef } \\
& \text { organe de prêhension } \\
& \text { obturateur }
\end{aligned}
$$$$
\text { avion, hélicoptère... }
$$$$
\text { benne, griffes, pince... }
$$$$
\text { clapet, soupape, opercule... }
$$

En somme, 11 faut savoir que ces niveaux de langue existent et en fouer selon le registre ou l'effet recherché.

LA LANGUE TECHNIQUE EST CHOSOCENTRIQUE

En technique, c'est bien connu, on ne s'intéresse qu'aux choses. Cette attitude se reflète notamment dans l'emplol du passif. Ce mode, plutôt décrié par les manuels de style, occupe dans les textes techntques une place assez importante. 
Souvent, par exemple, II n'est pas question de nommer l'agent: ce qui importe alors, c'est l'objet et l'action qu'il subit, et l'expression de 1 'agent devient alors une nuisance. Comme 11 est difficile de le supprimer comme sujet, on le supprime comme complément; l'objet bascule alors en position sujet, ce qui donne un passif:

La vapeur est infectée pendant toute la durée de 1' opération.

L'alimentation n'est couée automatiquement qu'en cas

d'urgence.

Dans ces deux exemples, l'agent n'est mème pas envisagê dans l'esprit du locuteur, au point qu'1l seralt fort douteux de remplacer le tour passif par la construction active avec on.

Mals on constate dans 1 'écrtture technique contemporaine une véritable soif de passif, même dans le cas où la construction avec on est possible. Témoin les deux exemples suivants:

Un vielllissement accélêré est souvent provoquể artificlellement par un traitement de stabilisation.

Les éléments sont obtenus dans un premier temps par sclage.

On constate une intention délibérée de mettre la chose en valeur: au début de la phrase et en position sujet. Le discours technique, on le remarque, a un point de vue fortement axé sur le mond des choses -- au détriment de celul des personnes. Et cette chosentricité se répercute effectivement sur les cholx syntaxiques.

On peut volr aussi dans ces deux exemples un simple solution de facilité consistant tout simplement à commencer la phrase par le premier mot qui vient à l'esprit (normalement celui considéré comme le plus important par le locuteur), ou encore un façon de s'exprimer caractéristique du monde technique moderne, terre-à-terre, pressé, désireux d'aller tout de suite au falt sans s'embarrasser de structures trop prévoyantes. Quoi qu'il en soit, on a affalre lci à une tendance stylistique avérée qui falt bel et bien partie du discours technique.
LA LANGUE TECHNIQUE SE PERMET DES ÉCARTS DE CONDUITE

La langue technique ne craint pas de rester en marge des critères de correction quí prévalent dans la langue générale. On pourralt aller jusqu'à dire que ces écarts de conduite contribuent à affirmer la personnalité propre de la langue technique.

Tours elliptiques

Par désır de concision, la langue technique entre parfois en conflit avec la grammatre normale:
220 volts monophasé

moyeu claveté coulissant sur bague montée dure sur

deux solutions soudées pour un bâti

un meuble laqué au four

le piston est échancré côté bielle descendante (accord avec 1e mot courant sous-entendu)

(attribut inopiné: emplo1 fréquent en technique)

(sans commentalre)

(sans commenta1re)

(du côté où se trouve 1 a bielle quand elle descend)
De telles tournures elliptiques, que plusieurs jugeralent effrontées (et qui sont inacceptables aux yeux de la grammatre actuelle), ne sont pas rares dans 1 'usage technique.

Enfin, signalons que la règle qui interdit de falre sulvre directement le pronom celui ou celle d'un participe est pratiquement tombée en désuétude en technique, sans doute pour des ratsons bien pragmatiques de brièvetê:

...en remplacement de celul fournı avec le modèle

standard.

\section{Règles formelles}

On constate une certalne résłstance à diverses règles formelles imposées de l'extérieur, notamment en ce qui concerne les unités de mesure et les abrévlations.

La règle qu1 oblige à n'employer le tralt oblique de division que pour les symboles (tr/min) et à employer par si les unités sont écrites au long (tours par minute) n'est que rarement respectée: on êcrit couramment tours/minute. Par allleurs, le tralt oblique falt 
partie d'un certain nombre de mors composés: rapport qualité/prix, échangeur eau/huile, etc., ce qui n'est pas admis par l'orthographe classique.

Bien que l'Académie des sciences ait banni ppm (parties par million) et proposé millionnieme, c'est bien ppm qui domine l'usage.

Les abréviations AV (avant) et AR (arrière), classiques en technique, vont à l'encontre des lols régissant les abréviations.

\section{Prépositions}

La langue technique tend à favoriser ses propres emplois prépositifs, notamment la préposition a:

$\begin{array}{ll}\text { inattaquable aux acides } & \text { (par les acides) } \\ \text { concentrique au tuyau } & \text { (par rapport au) } \\ \text { rigidité à la flexion } & \text { (vis-à-vis de la) }\end{array}$

Calques de vocabulaire

Parallèlement à l'usage de la langue générale, le vocabulaire de la langue technique puise aux langues étrangères (tout particulièrement

à 1 'anglais) pour falre ses propres emprunts et ses propres calques. DÉPRESSURISER

Il faut dépressuriser la bouteille avant d'enlever le robinet.

CLIPSER

Cette bavette se clipse et se déclipse facilement.

De même, 11 fait suivre à des mots courants une évolution non admise en langue générale:

POSITIF

La courrole crantée assure un entrafnement positif.

SÉVÈrE

Les conditions de travall de l'hulle sont sévères.

APPLICATION

Cette pompe se prête à des applications variées.

DÉLIVRER

Ce moteur délivre une puissance utille de...

\section{GËNÉRER}

Les signaux générés par le capteur sont mis en forme,

puis...

DËVELOPPER

Ce moteur développe $123 \mathrm{ch}$ à $3500 \mathrm{tr} / \mathrm{min}$.

\section{CONCLUSION}

On remarquera que dans ces traits généraux, la notion de style est absente. Pourtant, n'existerait-11 pas un style technique? On pense bien sûr au cas du manuel d'entretien, qui constitue une 81 grande part de la documentation technique. Mais ce serait par trop simplifler les choses. En réalité, il existe plusieurs styles en technique, selon qu'1l s'agit d'un article de vulgarisation, d'un mode d'emplo1, d'une annonce publicitalre... Et ces styles ne sont pas particuliers au technique; la manuel d'entretien, par exemple, se rapproche nettement du style administratif. C'est pourquol nous nous sommes limité à l'aspect de la langue elle-même, quil concerne tous les genres de textes techniques.

Les cours de perfectionnement en langue françalse que reçoit le rédacteur technique de formation académique sont axês sur 1a langue générale; à ce titre, ils peuvent 1 'amener à craindre les particularftés de la langue technique. Cet article vise justement à combler cette lacune, en lui montrant à quel point la langue technlque dispose de moyens d'expressions dynamiques et modernes, et aussi en montrant que ces particularités sont légltimes même si elles s'écartent de 1a langue générale. Souhaitons que l'enselgnement de la rédaction technique $s^{\prime}$ attarde davantage sur cette question à $I^{\prime}$ avenir.

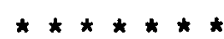

Claude Bédard dirfige sa propre entreprise:

$$
\begin{aligned}
& \text { Claude Bédard } \\
& \text { Technical Translation for Industry } \\
& 2705 \text { Edouard-Montpetit Boulevard } \\
& \text { Montréal., Québec }
\end{aligned}
$$$$
\text { H3T } 1 \mathrm{~J} 6
$$ 